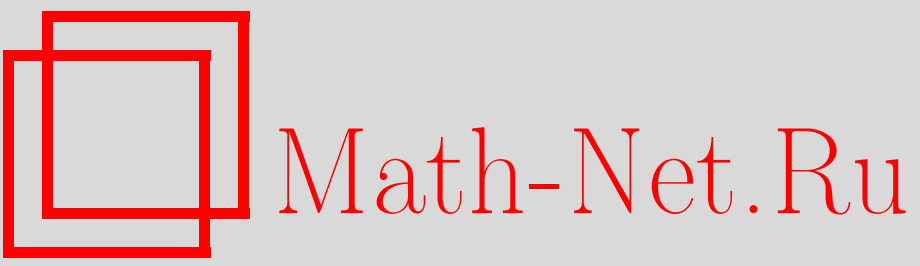

О. И. Мохов, Пара Лакса для неособых пучков метрик постоянной римановой кривизны, УМН, 2002, том 57, выпуск 3, 155-156

DOI: https://doi.org/10.4213/rm521

Использование Общероссийского математического портала Math-Net.Ru подразумевает, что вы прочитали и согласны с пользовательским соглашением

http://www.mathnet.ru/rus/agreement

Параметры загрузки:

IP : 3.82 .47 .9

26 апреля 2023 г., 13:44:53 


\title{
ПАРА ЛАКСА ДЛЯ НЕОСОБЫХ ПУЧКОВ МЕТРИК ПОСТОЯННОЙ РИМАНОВОЙ КРИВИЗНЫ
}

\author{
О.И. Мохов
}

Получены нелинейные уравнения, описьвающие все неособые пучки метрик постоянной римановой кривизны, и доказана интегрируемость этих уравнений методом обратной задачи (см. [1]). Для плоских пучков метрик соответствующие утверждения и доказательства были представлены в работах автора [2], [3], где предложен метод интегрирования нелинейных уравнений для неособых плоских пучков метрик. В [4] указана пара Лакса для неособых плоских пучков метрик. Эта пара Лакса обобщается на случай произволных неособых пучков метрик постоянной римановой кривизны. Задача описания пучков метрик постоянной римановой кривизны эквивалентна задаче описания согласованных нелокальных скобок Пуассона гидродинамического типа, порождаемых метриками постоянной римановой кривизны (согласованных скобок Мохова-Ферапонтова [5]). Две контравариантные метрики $g_{1}^{i j}(u)$ и $g_{2}^{i j}(u)$ постоянной римановой кривизны $K_{1}$ и $K_{2}$ соответственно назьваются согласованными, если любая линейная комбинация этих метрик $g^{i j}(u)=\lambda_{1} g_{1}^{i j}(u)+\lambda_{2} g_{2}^{i j}(u)$, где $\lambda_{1}$ и $\lambda_{2}$ - произволшные константы, для которых $\operatorname{det}\left(g^{i j}(u)\right) \not \equiv 0$, является метрикой постоянной римановой кривизны $\lambda_{1} K_{1}+\lambda_{2} K_{2}$, а коэфффициенты соответствующих связностей Леви-Чивиты связаны соотношением $\Gamma_{k}^{i j}(u)=\lambda_{1} \Gamma_{1, k}^{i j}(u)+$ $\lambda_{2} \Gamma_{2, k}^{i j}(u)[1]$ (метрики $g_{1}^{i j}(u)$ и $g_{2}^{i j}(u)$ образуют пучок метрик постоянной римановой кривизны [1]). Пара метрик $g_{1}^{i j}(u)$ и $g_{2}^{i j}(u)$ назьвается неособой, если собственные значения этой пары метрик, т.е. корни уравнения $\operatorname{det}\left(g_{1}^{i j}(u)-\lambda g_{2}^{i j}(u)\right)=0$, различны (пучок метрик, образованный неособой парой метрик, называется неособым). В [1] доказано, что для классификации всех неособых пучков метрик постоянной римановой кривизны достаточно классифицировать пары диагональных метрик постоянной римановой кривизны вида $g_{2}^{i j}(u)=g^{i}(u) \delta^{i j}$ и $g_{1}^{i j}(u)=f^{i}\left(u^{i}\right) g^{i}(u) \delta^{i j}$. Задача описания $N \times N$ диагональных метрик постоянной римановой кривизны $K$ эквивалентна задаче описания ортогональных криволинейных систем координат в $N$-мерном пространстве постоянной кривизны $K$. В [6] Захаров показал, что уравнения Ламе, описывающие ортогоналшные криволинейные системы координат в плоском пространстве, интегрируются методом обратной задачи. Аналогично интегрируются и уравнения, описьвающие ортогональные криволинейные системы координат в пространстве постоянной кривизны. Введем классические обозначения

$$
g^{i}(u)=\frac{\varepsilon^{i}}{\left(H_{i}(u)\right)^{2}}, \quad d s^{2}=\sum_{i=1}^{N} \varepsilon^{i}\left(H_{i}(u)\right)^{2}\left(d u^{i}\right)^{2}, \quad \beta_{i k}(u)=\frac{1}{H_{i}(u)} \frac{\partial H_{k}}{\partial u^{i}}, \quad i \neq k,
$$

где $H_{i}(u)$ - коэффициенты Ламе и $\beta_{i k}(u)-$ коэффициенты вращения, $\varepsilon^{i}= \pm 1, i=1, \ldots, N$.

Неособые пары согласованных метрик постоянной римановой кривизны $K_{1}$ и $K_{2}$ описываются следующими совместными интегрируемыми нелинейньми системами:

$$
\begin{gathered}
\frac{\partial \beta_{i j}}{\partial u^{k}}=\beta_{i k} \beta_{k j}, \quad i \neq j, \quad i \neq k, \quad j \neq k, \\
\varepsilon^{i} \frac{\partial \beta_{i j}}{\partial u^{i}}+\varepsilon^{j} \frac{\partial \beta_{j i}}{\partial u^{j}}+\sum_{s \neq i, s \neq j} \varepsilon^{s} \beta_{s i} \beta_{s j}=-K_{2} H_{i} H_{j}, \quad i \neq j, \\
\varepsilon^{i} f^{i}\left(u^{i}\right) \frac{\partial \beta_{i j}}{\partial u^{i}}+\frac{1}{2} \varepsilon^{i}\left(f^{i}\right)^{\prime} \beta_{i j}+\varepsilon^{j} f^{j}\left(u^{j}\right) \frac{\partial \beta_{j i}}{\partial u^{j}} \\
+\frac{1}{2} \varepsilon^{j}\left(f^{j}\right)^{\prime} \beta_{j i}+\sum_{s \neq i, s \neq j} \varepsilon^{s} f^{s}\left(u^{s}\right) \beta_{s i} \beta_{s j}=-K_{1} H_{i} H_{j}, \quad i \neq j,
\end{gathered}
$$

где $f^{i}\left(u^{i}\right), i=1, \ldots, N,-$ произвольные заданные функции одной переменной. Уравнения (2) - известные уравнения $n$-волн (уравнения Дарбу [7]), а уравнения (1)-(3) описывают ортого-

Работа вьполнена при финансовой поддержке Фонда Александра фон Гумболдта (Германия), а также РФФИ (грант № 02-01-00803) и фонда INTAS (грант № 99-1782). 
налњные криволинейные системш координат в $N$-мерном пространстве постоянной кривизны $K_{2}$, в частности, при $K_{2}=0$ получаются знаменитые уравнения Ламе. Система (1)-(4) совместна для любых функций $f^{i}\left(u^{i}\right)$, при этом общее решение зависит от $N^{2}$ произвольных функций одной переменной (функции $\beta_{i j}(u)$ и $H_{j}(u)$ могут быть заданы произвольно на $j$-й координатной линии). Пара Лакса для неособых плоских пучков метрик (при $K_{1}=K_{2}=0, \varepsilon^{i}=1$ ) указана в [4]:

$$
\frac{\partial \varphi_{i}}{\partial u^{j}}=\sqrt{\frac{\left(\lambda+f^{i}\right)}{\left(\lambda+f^{j}\right)}} \beta_{i j} \varphi_{j}, \quad i \neq j, \quad \frac{\partial \varphi_{i}}{\partial u^{i}}=-\sum_{k \neq i} \sqrt{\frac{\left(\lambda+f^{k}\right)}{\left(\lambda+f^{i}\right)}} \beta_{k i} \varphi_{k},
$$

где $\lambda$ - спектральный параметр. Линейная задача для уравнений Ламе была хорошо известна еще Дарбу [7], см. также, например, [6] (здесь $\varepsilon^{i}=1$ ):

$$
\frac{\partial \varphi_{i}}{\partial u^{j}}=\beta_{i j} \varphi_{j}, \quad i \neq j, \quad \frac{\partial \varphi_{i}}{\partial u^{i}}=-\sum_{k \neq i} \beta_{k i} \varphi_{k}
$$

Пару Лакса (5) легко получить из классической линейной задачи (6) для уравнений Ламе. Действительно, система уравнений, определяющая неособый плоский пучок метрик, получается из условия, что диагональная метрика $\left(\lambda+f^{i}\left(u^{i}\right)\right) g^{i}(u) \delta^{i j}$ является плоской при любом $\lambda$. Очевидно, что в этом случае (см. [2], [3]) в уравнениях Ламе необходимо заменить $\beta_{i j}(u)$ на $\widehat{\beta}_{i j}(u)=$ $\sqrt{\left(\lambda+f^{i}\left(u^{i}\right)\right) /\left(\lambda+f^{j}\left(u^{j}\right)\right)} \beta_{i j}(u)$. При этом линейная задача (6) переходит в пару Лакса (5). Пара Лакса (5) обобшается и на случай любых неособых пучков метрик постоянной римановой кривизны (1)-(4). Пара Лакса для системы (1)-(4) легко получается из линейной задачи для классической системы (1)-(3), описьвающей все ортогональные криволинейные системы координат в $N$-мерном пространстве постоянной кривизны $K_{2}$ :

$\frac{\partial \varphi_{i}}{\partial u^{i}}=-\sum_{k \neq i} \sqrt{\varepsilon^{k} / \varepsilon^{i}} \beta_{k i} \varphi_{k}+\sqrt{K_{2} / \varepsilon^{i}} H_{i} \psi, \frac{\partial \varphi_{i}}{\partial u^{j}}=\sqrt{\varepsilon^{i} / \varepsilon^{j}} \beta_{i j} \varphi_{j}, i \neq j, \frac{\partial \psi}{\partial u^{i}}=-\sqrt{K_{2} / \varepsilon^{i}} H_{i} \varphi_{i}$

(условие совместности системы (7) определяет уравнения (1)-(3)). Действительно, система уравнений (1)-(4) эквивалентна условию, что диагональная метрика $\left(\lambda+f^{i}\left(u^{i}\right)\right) g^{i}(u) \delta^{i j}$ является метрикой постоянной кривизны $\lambda K_{2}+K_{1}$ при любом $\lambda$. В этом случае (см. [2], [3]) в уравнениях (1)-(3) необходимо заменить $\varepsilon^{i}$ на $\epsilon^{i}, H_{i}(u)$ на $\widehat{H}_{i}(u)=H_{i}(u) / \sqrt{\varepsilon^{i} \epsilon^{i}\left(\lambda+f^{i}\left(u^{i}\right)\right)}, \beta_{i j}(u)$ на $\widehat{\beta}_{i j}(u)=\sqrt{\left(\varepsilon^{i} \epsilon^{i}\left(\lambda+f^{i}\left(u^{i}\right)\right)\right) /\left(\varepsilon^{j} \epsilon^{j}\left(\lambda+f^{j}\left(u^{j}\right)\right)\right)} \beta_{i j}(u), K_{2}$ на $\widehat{K}=\lambda K_{2}+K_{1}, \epsilon^{i}= \pm 1$. При этом линейная задача (7) переходит в пару Лакса

$$
\begin{gathered}
\frac{\partial \varphi_{i}}{\partial u^{i}}=-\sum_{k \neq i} \sqrt{\frac{\varepsilon^{k}\left(\lambda+f^{k}\right)}{\varepsilon^{i}\left(\lambda+f^{i}\right)}} \beta_{k i} \varphi_{k}+\sqrt{\frac{\lambda K_{2}+K_{1}}{\varepsilon^{i}\left(\lambda+f^{i}\right)}} H_{i} \psi, \\
\frac{\partial \varphi_{i}}{\partial u^{j}}=\sqrt{\frac{\varepsilon^{i}\left(\lambda+f^{i}\right)}{\varepsilon^{j}\left(\lambda+f^{j}\right)}} \beta_{i j} \varphi_{j}, \quad i \neq j, \quad \frac{\partial \psi}{\partial u^{i}}=-\sqrt{\frac{\lambda K_{2}+K_{1}}{\varepsilon^{i}\left(\lambda+f^{i}\right)}} H_{i} \varphi_{i},
\end{gathered}
$$

где $\lambda$ - спектральньй параметр. Условие совместности линейной системы (8), (9) эквивалентно уравнениям (1)-(4).

\section{СПИСОК ЛИТЕРАТУРЫ}

[1] О. И. Мохов // Функц. анализ и его прил. 2001. Т. 35. № 2. С. 24-36; math.DG/0005051. [2] O. I. Mokhov // math.DG/0005081. [3] О. И. Мохов // УМН. 2001. Т. 56. № 2. С. 221-222. [4] E. V. Ferapontov // math.DG/0005221. [5] О. И. Мохов, Е. В. Ферапонтов // УМН. 1990. T. 45. № 3. C. 191-192. [6] V. E. Zakharov // Duke Math. J. 1998. V. 94. № 1. P. 103-139. [7] G. Darboux. Leçons sur les systèmes orthogonaux et les coordonnées curvilignes. Paris: Gauthier-Villars, 1910.

Центр нелинейных исследований при Институте теоретической физики им. Л. Д. Ландау РАН 